\title{
Commentary
}

\section{Ophthalmic medical assistants: response}

The commentary on ophthalmic medical assistants (OMAs) by Webber and Jeffrey ${ }^{1}$ opens up an important topic. We recall a suggestion made a few years ago to create a new technical post to replace many of the duties normally undertaken by nurses and orthoptists. This generated much discussion but the issue eventually subsided leaving the status quo. The health service climate is now very different so this new proposal for the widespread introduction of OMAs needs to be given every consideration.

The job description of the OMA includes "cataract preoperative counselling, clerking, biometry measurements, compilation of operating lists, postoperative examinations, and even discharge from the clinic ... (and) can include all other aspects of ophthalmology." This is an impressive range of tasks especially for one with such a basic level of training, and certainly both trivialises and seriously underestimates the depth and breath of ophthalmic care patients deserve to receive. With the OMA on board one wonders what is left for anyone else to do? It also begs the question of whether ophthalmologists are merely surgical technicians and do not need to be medically qualified? The authors frequently compare UK and US ophthalmology, a limited analogy as the two health systems differ so greatly.

According to the authors a key feature is the one to one relationship between OMA and ophthalmic surgeon. This is fine for mobile phones, which do not require much in the way of breaks for meals, holidays, or leave for professional development, and are even cheaper after hours and at weekends. But is it appropriate for humans? Is there an ophthalmic marriage broker who can match perfectly one OMA with one ophthalmologist until death (or retirement) do them part? Indeed, is this a desirable working relationship, and is it sustainable in the long term? Webber and Jeffrey state that one to one improves communication and bypasses the "restrictive rules and regulations", which they say govern nursing practice, and allows the OMA to "be able to carry out any task his or her consultant requests". The mind boggles - is this impeachable? Such an odd mixture of patriarchy and old fashioned "union speak". Can they be so naive that they do not recognise that OMAs will naturally form or join a professional organisation which will properly produce rules and regulations. One of the first tasks of such a body inevitably will be to break down the one to one relationship to ensure for the OMA a greater breadth of experience, and a more healthy working atmosphere than is possible with one (subspecialised) ophthalmologist-back to square one! Patients, like professionals, need to be protected and they require to be reassured that they will receive a uniform quality of care across the country. This is achieved by nationally regulated bodies through the mechanism of state registration which ensures the good training and conduct of professionals. This is costly and time consuming to set up, but already exists for orthoptists, nurses, and optometrists.

Webber and Jeffrey focus mainly on the surgical aspects, but ophthalmic care is both surgical and medical and also covers a wide range of acute and chronically disabling con- ditions. Thankfully, the divide between hospital and the rest of the community is being eroded and increasingly we are required to work both within and without the hospital setting. Ophthalmologists and nurses get a mention, ${ }^{1}$ but not other members of the ophthalmic team such as orthoptists, optometrists, and a range of technical grades - an important omission, as the roles of individual team members vary according to local needs, enthusiasm, and prejudices. It is not at all surprising, as with any active team, that there is some jostling and discussion. This is a pain and all too often pretty uncomfortable, but it is healthy and laudable, and it is the stuff of progress. It is quite distinct from the one to one ophthalmologist-OMA relationship, in which all the running must come from the ophthalmologist. One of the immeasurable and broadening benefits of teamwork is that each profession, coming as it does from a different background, brings its own unique and complementary skills. When it comes to public perception of hands-on care and communication nobody does it better than nurses; similarly orthoptists have unparalleled patience and expertise in dealing with babies and children. Do we really want to throw this away? The OMA as described here ${ }^{1}$ is very specific-task oriented, a stark distinction from the more holistic approach appropriate to a professional.

So much for criticism. Webber and Jeffrey should be congratulated for raising this critical issue: currently the ophthalmic team does not function as well as it might. It is rather inflexible and does not adapt speedily enough to meet the needs of modern ophthalmology. There are gaps and inefficiencies. The OMA, with its undoubted innovative features, was created to overcome these problems, but it has not been fully thought through and does not encompass the full spectrum of ophthalmic care. It is a short term fix which we believe cannot be sustained in the long term; and most of all it fails to address the fundamental issues. It makes no sense at all to disguise as an OMA the very person who was considered to function unsatisfactorily as a nurse or orthoptist.

Now is the time to reappraise and modify the function of the ophthalmic team, and the roles of its constituents, so that we work better together to provide comprehensive, efficient, and effective care. Above all, this is not the time to confuse the picture further by creating yet another profession allied to ophthalmology-a sort of sycophantic ophthalmic butler-which will also in time become largely obsolete.

ALISTAIR FIELDER

Ophthalmologist, Academic Unit of Ophthalmology,

Imperial College School of Medicine, Western Eye Hospital, London NW1 5YE

HELEN POINTER

Nurse manager, Mayday University Hospital NHS Trust, Croydon CHRIS TIMMS

Senior orthoptist, Great Ormond Street Hospital for Children NHS Trust, London

1 Webber SK, Jeffrey MN. Ophthalmic medical assistants. Br f Ophthalmol 1999;83:4. 